\title{
Modulation, Bioinformatic Screening, and Assessment of Small Molecular Peptides Targeting the Vascular Endothelial Growth Factor Receptor
}

\author{
Shibin Feng $\cdot$ Lingyun Zou $\cdot$ Qingshan Ni $\cdot$ \\ Xiang Zhang $\cdot$ Qianwei Li $\cdot$ Lei Zheng $\cdot$ \\ Laiping Xie $\cdot$ Hongmin Li $\cdot$ Dingde Huang
}

Published online: 29 July 2014

(c) The Author(s) 2014. This article is published with open access at Springerlink.com

\begin{abstract}
Vascular endothelial growth factor (VEGF) and VEGF receptor (VEGFR) are important factors in tumor growth and metastasis. Molecular probes or drugs designed to target VEGF/VEGFR interactions are crucial in tumor molecular imaging and targeted therapy. Bioinformatic methods enable molecular design based on the structure of bio-macromolecules and their interactions. This study was aimed to identify tumor-targeting small-molecule peptides with high affinity for VEGFR using bioinformatics screening. The VEGFR extracellular immunoglobulin-like modules Ig1-Ig3 were used as the target to systematically alter the primary peptide sequence of $\mathrm{VEGF}_{125-136}$. Molecular docking and surface functional group interaction methods were combined in an in silico screen for polypeptides, which in theory, would have higher affinities for VEGFR. In vitro receptor competition binding assays were used to assess the affinity of the putative VEGFR-binding polypeptides. Rhodamine-conjugated peptides were used to label and visualize peptide-binding sites on A549 cells. Using bioinformatic screening, we identified 20 polypeptides with
\end{abstract}

Shibin Feng and Lingyun Zou are Co-first authors.

S. Feng - X. Zhang - Q. Li - L. Zheng - L. Xie · H. Li ·

D. Huang $(\bowtie)$

Department of Nuclear Medicine, Southwest Hospital, Third

Military Medical University, 30 Gaotanyan Street, Shapingba

District, Chongqing 400038, China

e-mail: huangdde@tmmu.edu.cn

S. Feng

e-mail: wlf1211@126.com

\section{Zou $\cdot$ Q. Ni}

Department of Microbiology, College of Basic Medical

Sciences, Third Military Medical University, 30 Gaotanyan

Street, Shapingba District, Chongqing 400038, China

e-mail: lyzou@tmmu.edu.cn potentially higher affinity for VEGFR. The polypeptides were capable of inhibiting the binding of ${ }^{125} \mathrm{I}$-VEGF to VEGFR in a dose-dependent manner. The $\mathrm{IC}_{50}$ values of QKRKRKKSRKKH and RKRKRKKSRYIVLS (80 and $185 \mathrm{nmol} / \mathrm{L}$, respectively) were significantly lower than that of $\mathrm{VEGF}_{125-136}(464 \mathrm{nmol} / \mathrm{L})$; thus, the affinity of these peptides for VEGFR was 6- and 2.5-fold higher, respectively, than that of $\mathrm{VEGF}_{125-136}$. Rhodamine labeling of A549 cells revealed peptide binding mainly on the plasma membrane and in the cytoplasm. Bioinformatic approaches hold promise for the development of molecular imaging probes. Using this approach, we designed two peptides that showed higher affinity toward VEGFR. These polypeptides may be used as molecular probes or drugs targeting VEGFR, which can be utilized in molecular imaging and targeted therapy of certain tumors.

Keywords Bioinformatics - Molecular probe $\cdot$ Tumor * VEGF/VEGFR

\section{Introduction}

In tumor diagnosis and therapy, the discovery of diseaserelevant molecular targets and the construction of molecular probes or targeted drugs with high specificity for these targets are crucial $[1,2]$. Certain molecules, including vascular endothelial growth factor receptor (VEGFR), integrin $\alpha_{v} \beta_{3}$ [3, 4], somatostatin receptor [5], vasoactive intestinal peptide receptor [6], matrix metalloproteinases [7], E-selectin [8], and CD105 [9], are expressed at higher levels in tumor cells and in newly formed vascular endothelial cells. Thus, these molecules are often used as targets in tumor-targeted radionuclide imaging or therapy [10]. Among these molecules, vascular endothelial growth factor 
(VEGF) is the principal factor mediating tumor growth, angiogenesis, and metastasis. Molecular probes or targeted drugs based on VEGF or VEGFR can be widely applied in tumor-targeted molecular imaging or therapy [11-17].

VEGF $_{125-136}$ is a 12-amino acid peptide (QKRKRK KSRYKS) encoded by the VEGF-A gene (exon 6). VEGF $_{125-136}$ binds specifically to VEGFR, but does not activate its signal pathway $[18,19]$. Our previous study demonstrated that VEGF $_{125-136}$ exhibits good tumor-targeting properties, and could be used as a highly specific agent for tumor radionuclide imaging and therapy [20]. However, the previous in vitro study of $\mathrm{VEGF}_{125-136}$ suggested that it has a relatively weak capacity for tumor growth inhibition and exhibits a relatively short retention time in tumor tissues. Molecular probes and tumor-targeting drugs need to possess high binding affinity as well as a long half-life in the tumor tissues; hence, we aimed to modify VEGF $_{125-136}$ to improve its affinity for VEGFR.

The current study utilized a combination of bioinformatics and in vitro experimentation to improve $\mathrm{VEGF}_{125-136}$. Two polypeptides with higher VEGFR-binding affinity were selected from the peptide candidates, which may be used as molecular probes or targeted drugs.

\section{Materials and Methods}

Analysis of Molecular Docking and Surface Functional Group Interaction

\section{Experimental Platform}

The analyses were performed using the high-performance computing system at the Bioinformatics Center of Third Military Medical University (Chongqing, China), which is based on the open 64-bit Linux parallel computing system Rocks Cluster 5.3 (http://www.rocksclusters.org/). The system consists of 26 dual quad-core servers ( 208 computing cores) and is capable of $\approx 2$ TFLOPS. The scheme used for bioinformatic screening of peptides with high VEGFRbinding affinity is shown in Fig. 1. AutoDock-Vina 1.1 software was used for molecular docking analysis [21], while
Fig. 1 Scheme of bioinformatic screen for peptides with elevated VEGFR-binding affinity. a Peptide sequences are generated by manual design (here, the peptide "QKRKRKKSRKKH" is used as an example); $\mathbf{b}$ the initial structure of each peptide is predicted using PEP-FOLD software; c a stable peptide structure is generated by molecular dynamics simulation using NAMD (5000 steps of energy minimization and 500-ps dynamics simulation); $\mathbf{d}$ a docking procedure to VEGFR is performed for each peptide using AutoDock Vina software and scores assigned according to the output energy (here, the docking site between VEGR and "QKRKRKKSRKKH" with minimal energy output is shown); e sub-sequences cut from the peptide with a slidingwindow method used to predict binding specificity using the PEPSITE algorithm, are scored using Eq. (1), and the total binding score $S$ is calculated using Eq. (2) (the residues of the sub-sequences from

"QKRKRKKSRKKH" are shown as spheres binding to the $\operatorname{Ig} 1-\operatorname{Ig} 3$ domains of VEGR)
Example: QKRKRKKSRKKH

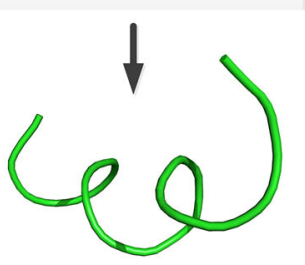

B: structure prediction by PEP-FOLD

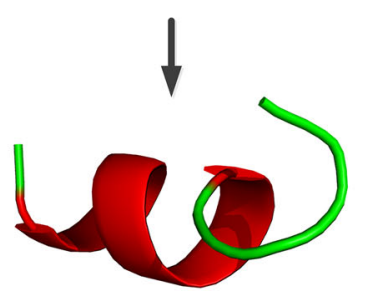

C: energy minimization and dynamic simulation by NAMD

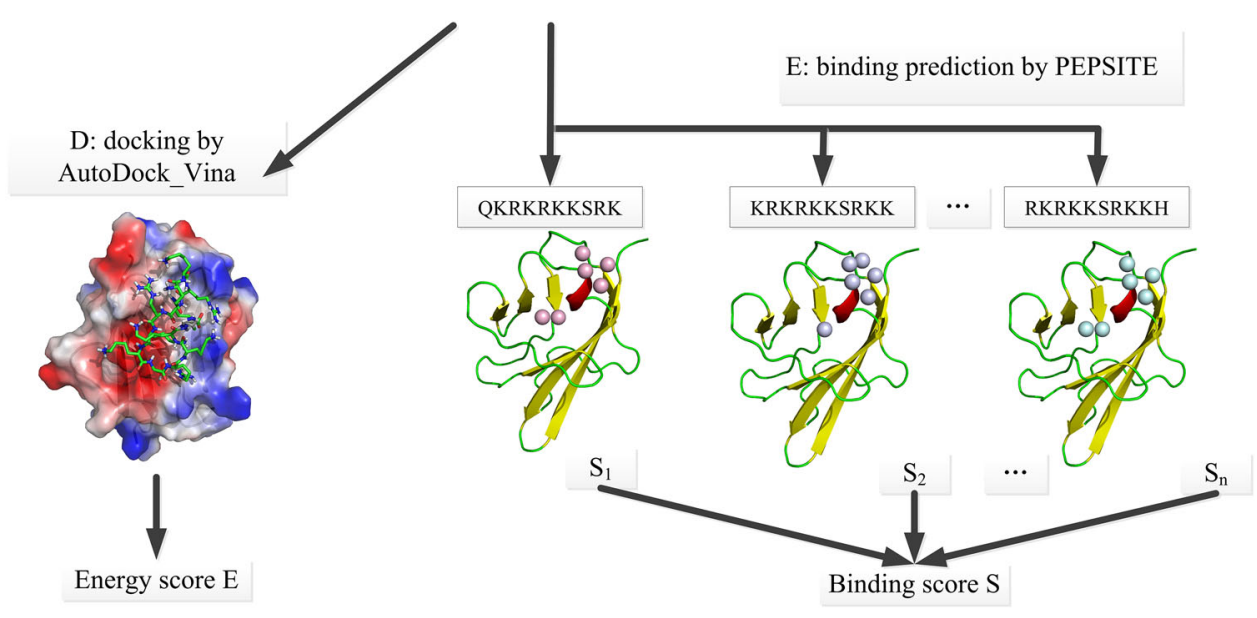


PyMol and the PEPSITE program [22] were used for surface functional group interaction analysis and prediction.

\section{Molecular Docking-Based Screening for Polypeptides with Enhanced VEGFR Affinity}

Based on the "lock-and-key" principle of the interaction between ligands and receptors, molecular docking methods simulate the interaction between a small-molecule ligand and a macromolecular receptor. The extracellular immunoglobulinlike modules Ig1-Ig3 of VEGFR reside in the ligand-binding domain and appear to form a rigid structure, whereas the ligand was observed to have a flexible structure. In our docking computation, we assumed that altered polypeptides based on VEGF $_{125-136}$ would interact with VEGFR in the same region. The binding energy of VEGFR and the polypeptides was calculated using AutoDock-Vina software, where lower binding energy indicates higher affinity interactions.

The initial approximate structure of $\mathrm{VEGF}_{125-136}$ was predicted by PEP-FOLD (http://bioserv.rpbs.univ-parisdiderot.fr/PEP-FOLD/), and NAMD (version 2.7, http:// www.ks.uiuc.edu/Research/namd/) was employed in the molecular dynamics simulation to obtain a refined structure. During molecular dynamics simulations, all peptide atoms were surrounded with a cubic water box of simple pointcharge water molecules that extended $10 \AA$ from the protein, and periodic boundary conditions were applied in all directions. The systems were neutralized with $\mathrm{Na}^{+}$and $\mathrm{Cl}^{-}$ counter ions replacing the water molecules, and a 5,000 step energy minimization was performed, followed by a 500-ps production molecular dynamics simulation with a time-step of 2 fs at constant pressure (1 atm) and temperature (300 K).

First, the binding energy of $\mathrm{VEGF}_{125-136}$ to VEGFR was calculated and used as the reference free energy. Then, leaving the core motif RKRKKSR of VEGF $125-136$ unaltered, we sequentially mutated the amino acids at the 1st, 2nd, 10th, 11th, and 12th positions of the sequence. The total number of mutations was $20 \times 20 \times 20 \times 20 \times$ $20=3.2 \times 10^{6}$. A hill-climbing algorithm was adopted to reduce the search range in calculating the molecular docking configuration and free binding energy between each mutated polypeptide and the receptor, and finally to screen for peptide sequences with lower binding energy.

\section{Surface Functional Group Interaction Analysis Based on VEGF $125-136$}

Studies have shown that the principle underlying the bonding between VEGF and VEGFR is based on ionic bonds and hydrophobic effects. Using PyMol, we analyzed the detailed interaction between VEGF and VEGFR (shown in Fig. 2). The surface of the peptide consists of numerous basic groups, resulting in strong interactions with the acidic groups in the extracellular domain of VEGFR. We also predicted the binding sites of "RKRKKSR" core motif on the extracellular Ig1-Ig3 domains of VEGFR using PEPSITE program. The results show that six residues of the core motif are apt to bind near to the Ig2 domain and matched well with the structure of the motif (shown in Fig. 3b), which enables the PEPSITE algorithm to accurately predict the binding sites of peptides on protein surfaces. PyMol analysis of the distribution of the extracellular Ig1-Ig3 domain surface groups revealed that hydrophobic pockets existed in proximity to multiple acidic groups (Fig. 3a). Therefore, we postulated that the fundamental approach in modulating surface group interaction should involve adding more basic hydrophilic amino acids to the VEGF $_{125-136}$ sequence, thereby increasing the interactions with acidic groups on the surface of VEGFR. Moreover, we added hydrophobic fragments to the termini of the polypeptide to achieve a stronger interaction with the hydrophobic pockets surrounding the acidic groups. Subsequently, the bonding specificity between the newly designed peptide sequences and the receptor surface was predicting using PEPSITE, and peptide sequences that bound to VEGFR with low $p$ values were obtained. Moreover, we developed a scoring scheme based on the PEPSITE prediction results. For the PEPSITE prediction, a window (length $L \leq 10$ residues) was slid on the peptide sequence, and several sub-sequences with $L$ residues were obtained (peptide length is limited to ten residues in PEPSITE). Each sub-sequence was used to predict binding specificity. The top ten results were employed to calculate binding scores $(S)$ for the sub-sequences, which were determined using the following equation:

$S=\sum_{i=1}^{n}\left(\frac{1}{p_{i}}\right)$,

where $n$ is the number of all top binding sites ( $n$ is 10 in this study); $p_{i}$ is the $p$ value of binding site $i$ of the top $n$ outputs by the PEPSITE algorithm. The total score $S^{\text {total }}$ for a peptide was calculated using the following equation:

$S^{\text {total }}=\frac{1}{m} \sum_{k=1}^{m}\left(S_{k}^{\text {sub }}\left(1+\log \frac{S_{k}^{\text {sub }}}{S^{\text {core }}}\right)\right)$,

where $m$ is the number of all sub-sequences; $S_{k}^{\text {sub }}$ and $S^{\text {core }}$ are the binding scores for sub-sequence $k$ and that of the core "RKRKKSR" motif calculated using Eq. (1), respectively.

\section{Evaluation of Polypeptides' Binding Affinity for VEGFR Using In Vitro Receptor Binding Assays}

A549 cells were cultured in 24-well culture plates at $3 \times 10^{4}$ cells/well in duplicate for each treatment. Cultures were maintained at $37{ }^{\circ} \mathrm{C}$ in a $5 \% \mathrm{CO}_{2}$ incubator. After $24 \mathrm{~h}$, the culture supernatant was discarded, and cells were washed in PBS, followed by the addition of serum-free culture medium 


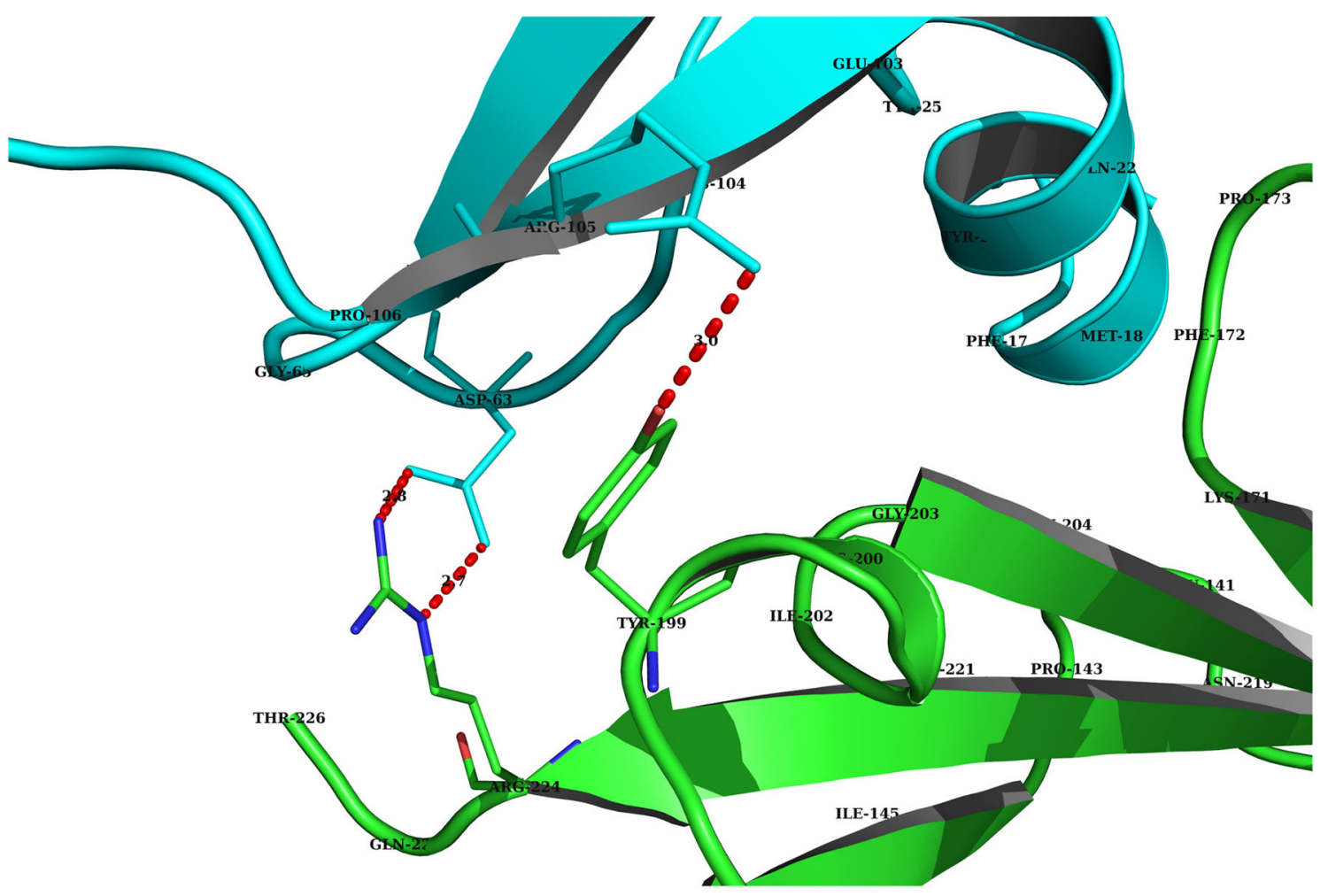

Fig. 2 Binding of VEGF and VEGFR as analyzed with PyMol. This interaction view was created using the dimer complex structure of VEGF and VEGFR (PDB id: 1FLT). The molecular structure is shown as a cartoon with green indicating the extracellular domain of VEGFR (chain $X$ ) and cyan indicating VEGF (chain W). All of the

(Hyclone, Logan, UT) before performing an in vitro competitive-binding assay. To each well, VEGF $_{125-136}$ or optimized peptides were added at the following concentrations: $0,1.3,6.5,32.5,65,650$, and $6,500 \mathrm{nmol} / \mathrm{L}$. After a 30 -min incubation period at $4{ }^{\circ} \mathrm{C}, 1.85 \mathrm{kBq}{ }^{125} \mathrm{I}_{-V^{-}} \mathrm{VGF}_{165}$ (PerkinElmer,Boston, MA) at a final concentration of $0.37 \mu \mathrm{g} / \mathrm{L}$ was added to each well, and the plates were further incubated for another $2 \mathrm{~h}$ at $4{ }^{\circ} \mathrm{C}$. The culture supernatant was discarded, and the cells were washed twice with pre-cooled $\left(4{ }^{\circ} \mathrm{C}\right)$ PBS containing $0.1 \%$ BSA to remove unbound ${ }^{125} \mathrm{I}_{-V^{\circ}} \mathrm{VGF}_{165}$. The cells were then trypsin digested and quickly filtered through glass fiber filter paper using a multi-channel microfluidic device. Radioactive counts (cpm) on the filter paper were determined using a $\gamma$-counter (Chongqing Optical \& Electrical Instrument). Peptide competition binding curves were plotted, and $\mathrm{IC}_{50}$ values for each peptide were calculated using SPSS13.0 statistics software.

\section{Identification of Peptide-Binding Sites on A549 Cells Using Fluorescence Microscopy}

The highest affinity peptides, NO.15 (QKRKRKKSRKKH) and NO.17 (RKRKRKKSRYIVLS), were conjugated with a fluorochrome, and fluorescence microscopy was used to residues within a distance of $<5 \AA$ between VEGF and the receptor have been labeled. All possible hydrogen bonds with a distance of $<3.2 \AA$ between VEGF and the receptor are shown as red dotted lines, where their lengths are calculated and labeled (Color figure online)

observe binding to A549 cells as follows. A549 cells were plated on coverslips and grown overnight. The cells were then washed three times with PBS and fixed with $4 \%$ paraformaldehyde for $15 \mathrm{~min}$ at room temperature. The cells were incubated at $37{ }^{\circ} \mathrm{C}$ for 30 min with $2 \%$ BSA to block nonspecific binding, and subsequently washed three times with PBS. Rhodamine-conjugated peptides $(45 \mu \mathrm{mol} / \mathrm{L})$ were added and incubated with the cells for $2 \mathrm{~h}$ at $4{ }^{\circ} \mathrm{C}$. After three washes with PBS, fluorescence microscopy (maximum absorption wavelength of $552 \mathrm{~nm}$ ) was used to observe cellular staining. Unconjugated rhodamine was used as staining control.

\section{Results}

Analysis of Molecular Docking and Surface Functional Group Interactions

Screening for Polypeptides with High Affinity for VEGFR Using Molecular Docking Analysis

Simulated VEGF $_{125-136}$ was docked semi-flexibly with VEGFR using AutoDock-Vina. The free binding energy was calculated five times, and the lowest binding energy 
Table 1 Peptide sequences with VEGFR-binding energy lower than $-5.2 \mathrm{kcal} / \mathrm{mol}$ (according to molecular docking)

\begin{tabular}{lll}
\hline Number & Sequence & Binding energy $(\mathrm{kcal} / \mathrm{mol})$ \\
\hline 1 & QFRKRKKSRYPK & -6.2 \\
2 & QKRKRKKSRYPK & -6.0 \\
3 & QFRKRKKSRYPS & -5.9 \\
4 & QKRKRKKSRYKY & -5.8 \\
5 & QFRKRKKSRWKS & -5.6 \\
6 & QKRKRKKSRYKK & -5.5 \\
7 & QFRKRKKSRYKS & -5.4 \\
8 & KFRKRKKSRYKS & -5.4 \\
9 & FFRKRKKSRYKS & -5.3 \\
10 & QFRKRKKSRYKH & -5.3 \\
11 & HFRKRKKSRYKS & -5.3 \\
12 & QFRKRKKSRYLS & -5.3 \\
13 & QFRKRKKSRYKF & -5.2 \\
14 & QFRKRKKSRYKP & -5.2 \\
15 & QFRKRKKSRYKQ & -5.2 \\
16 & DKRKRKKSRYKS & -5.2 \\
17 & QKRKRKKSRYWS & -5.2 \\
VEGF $125-136$ & QKRKRKKSRYKS & -5.0 \\
\hline
\end{tabular}

A

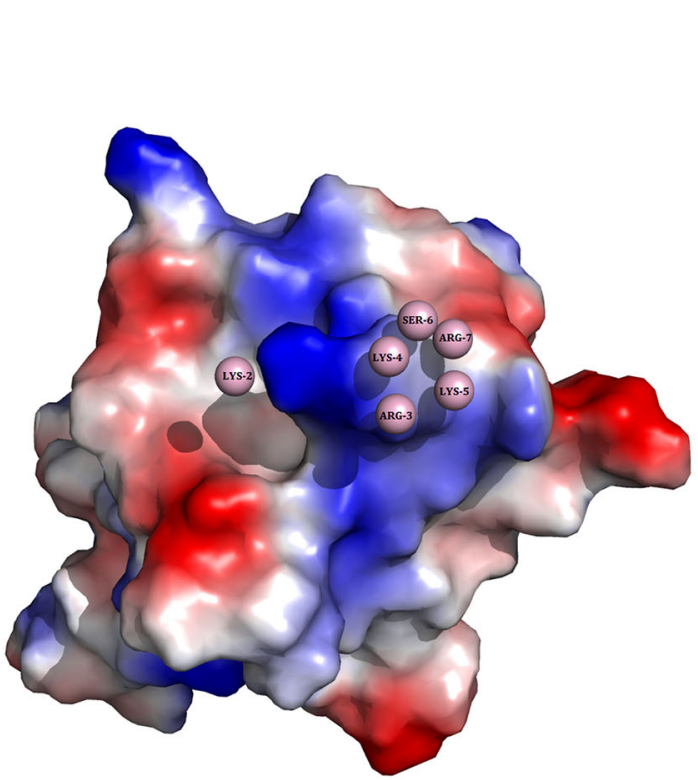

Fig. 3 Predicted binding sites of "RKRRKSR" core motif on the surface of VEGFR. The binding sites of "RKRKKSR" core motif on the VEGFR surface as predicted by the PEPSITE program. The six balls indicate the predicted locations of six residues from "RKRRKSR". a distribution of surface polar (white), acidic (red), basic (blue), and non-polar (white) groups in the extracellular domains of VEGFR. $\mathbf{b}$ the PEPSITE-binding pattern of the core motif and the extracellular score $(-5.0 \mathrm{kcal} / \mathrm{mol})$ was considered as the affinity score for the interaction between $\mathrm{VEGF}_{125-136}$ and VEGFR. In all, we identified 17 polypeptides with binding energy lower than $-5.2 \mathrm{kcal} / \mathrm{mol}$ using a hill-climbing algorithm (Table 1).

Screening of Polypeptides with Higher Affinity for VEGFR Using Surface Group Interaction Analysis

We predicted the binding region of "RKRKKSR" motif to be on the surface of extracellular domains of VEGFR using PEPSITE (Fig. 3a, b). The predicted sites are consistent with experimental results reported in the literature, and are well matched with the residue positions in the structure of the peptide. This provided important information with respect to the binding location of the core motif on the receptor. This was helpful in peptide design because the segment containing the core motif was absent in the crystal complex of VEGF and VEGFR.

We retained the core sequence (RKRKKSR) of VEGF $_{125-136}$ while gradually adding hydrophilic basic amino acids ( $\mathrm{R}, \mathrm{K}$, and $\mathrm{H})$ at the $\mathrm{N}$ - and $\mathrm{C}$-terminus of the

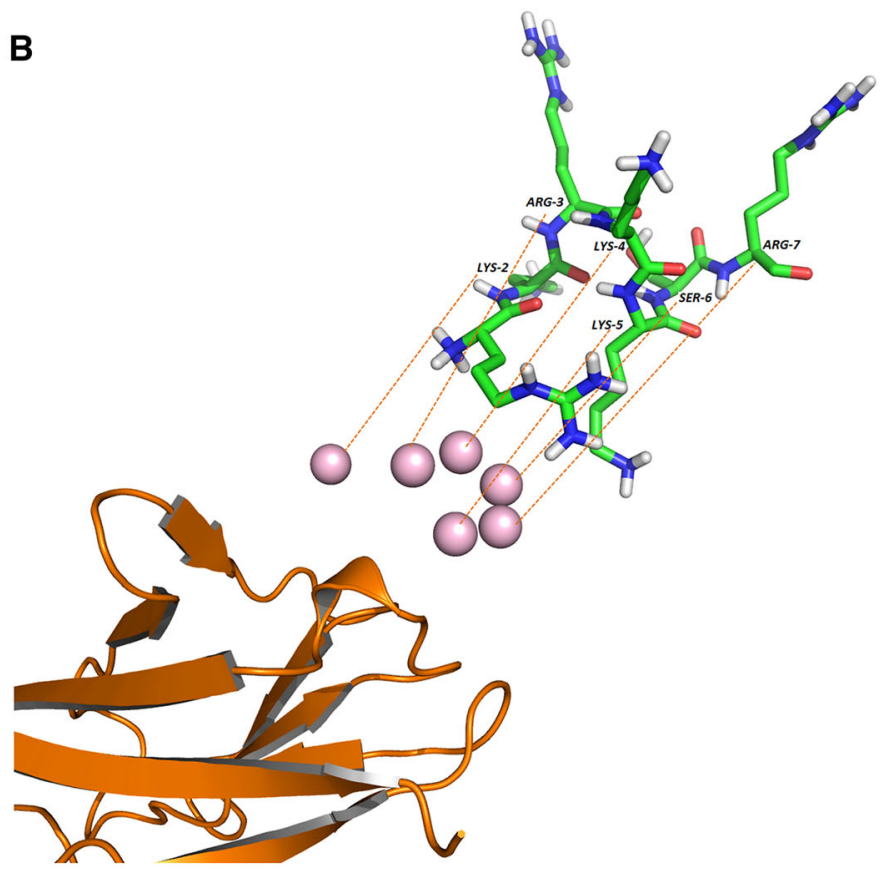

domains of VEGFR ( orange: extracellular Ig1-Ig3 domains of VEGFR, namely, chain $\mathrm{X}$ of VEGFR from the complex crystal structure of VEGF and VEGFR). The structure of the core motif predicted by PEPFOLD and simulated by NAMD is shown as sticks in the figure. Each binding residue is linked with the same residue in the structure using dotted lines. The image shows that residues in the core peptide matched well with the predicted binding sites (Color figure online) 
core sequence; alternatively, we replaced specific amino acids in the VEGF $_{125-136}$ sequence with hydrophilic basic amino acids. Furthermore, we added a certain number of basic amino acids to the core peptide, keeping the maximum length at 14 residues. Strong hydrophobic amino acids (I, L, V, and F) were added either at the $\mathrm{N}$ - or C-terminus of the sequence. A novel scoring scheme, based on the PEPSITE output, was adopted to produce the peptide-binding score on the receptor protein surface (Eqs. 1 and 2), with higher scores indicating stronger binding (Tables 2 and 3).

Thus, we selected 20 peptides with predicted outcomes better than VEGF $_{125-136}$ as candidate sequences (Table 4).

Evaluation of Polypeptides' Binding Affinity for VEGFR

All 20 selected peptides produced potent dose-dependent inhibition of ${ }^{125}$ I-VEGF binding to VEGFR, which

Table 2 Effect of adding basic groups to the $\mathrm{VEGF}_{125-136}$ sequence on PEPSITE score

\begin{tabular}{lll}
\hline Number & Sequence & $\begin{array}{l}\text { Score calculated in modified } \\
\text { PEPSITE (integrated score } S \text { ) }\end{array}$ \\
\hline 1 & QKRKRKKSRKKH & 36.8 \\
2 & RKRKRKKSRKKH & 36.8 \\
3 & KKRKRKKSRKRK & 36.2 \\
4 & QKRKRKKSRYRK & 35.6 \\
5 & QHRKRKKSRKRH & 34.9 \\
6 & RKRKRKKSRYKK & 34.7 \\
7 & QKRKRKKSRYKK & 30.8 \\
8 & QKRKRKKSRRKK & 29.5 \\
9 & QKRKRKKSRKK & 28.8 \\
10 & QKRKRKKSRHKK & 28.4 \\
VEGF $_{125-126}$ & QKRKRKKSRYKS & 27.5 \\
\hline
\end{tabular}

Table 3 Effect of adding hydrophobic groups to the $\mathrm{VEGF}_{125-136}$ sequence on PEPSITE score

\begin{tabular}{lll}
\hline Number & Sequence & $\begin{array}{l}\text { Score calculated in modified } \\
\text { PEPSITE (integrated score } S \text { ) }\end{array}$ \\
\hline 1 & QHKRKRKKSRIVL & 30.3 \\
2 & KFRKRKKSRYIV & 29.6 \\
3 & RKRKRKKSRYIVLS & 28.8 \\
4 & IVFKRKRKKSRYLS & 28.6 \\
5 & RKRKRKKSRKIVL & 27.1 \\
6 & ILLVRKRKSRYKK & 25.9 \\
7 & IVVRKRKSRYKH & 25.4 \\
8 & IVVRKRKSRYRK & 25.1 \\
9 & ILIVRKRKSRYKK & 24.3 \\
10 & RKRKKSRKKHIL & 24.1 \\
VEGF $_{125-136}$ & QKRKRKKSRYKS & 23.7 \\
\hline
\end{tabular}

suggests that all 20 peptides bound specifically to VEGFR. Thirteen polypeptides exhibited $\mathrm{IC}_{50}$ values lower than VEGF $_{125-136}(464 \mathrm{nmol} / \mathrm{L}$, Fig. $4 \mathrm{a})$, with the $\mathrm{IC}_{50}$ values of the remaining polypeptides exceeded this value. The $\mathrm{IC}_{50}$ values of QKRKRKKSRKKH and RKRKRKKSRYIVLS were significantly lower than that of $\mathrm{VEGF}_{125-136}$, at $80 \mathrm{nmol} / \mathrm{L}$ (Fig. 4b) and $185 \mathrm{nmol} / \mathrm{L}$ (Fig. 4c), respectively (Table 4). This corresponds to 6- and 2.5-fold increases in affinity for QKRKRKKSRKKH and RKRKRKKSRYIVLS, respectively. Blast searches of the NCBI database revealed that both peptides were novel.

Evaluation of Peptides Binding in A549 Cells Using Fluorescence Microscopy

The binding of rhodamine-conjugated peptides is shown in Fig. 5. After incubation with the A549 cells for $6 \mathrm{~h}$, specific labeling was observed using fluorescence microscopy. In contrast, incubation with unconjugated rhodamine did not result significant levels of fluorescent staining of A549 cells. Analysis of the merged bright field and fluorescent images demonstrated that specific binding of peptides NO.15 (QKRKRKKSRKKH) and NO.17 (RKRKRKK SRYIVLS) is observed in the plasma membrane and perinuclear cytoplasm of A549 cells.

Table 4 Twenty peptides with theoretical VEGFR-binding affinities exceeding that of $\mathrm{VEGF}_{125-136}$ and $\mathrm{IC}_{50}$ values from competitive VEGFR binding with ${ }^{125}$ I-VEGF

\begin{tabular}{llc}
\hline Number & Sequence & IC50 (nmol/L) \\
\hline Control & VEGF $_{125-136}$ & $464 \pm 12$ \\
15 & QKRKRKKSRKKH & $80 \pm 7$ \\
5 & QFRKRKKSRWKS & $92 \pm 9$ \\
17 & RKRKRKKSRYIVLS & $185 \pm 14$ \\
10 & FFRKRKKSRYKS & $188 \pm 25$ \\
11 & QFRKRKKSRYKH & $252 \pm 16$ \\
1 & QFRKRKKSRYPK & $273 \pm 19$ \\
9 & KFRKRKKSRYIV & $273 \pm 33$ \\
4 & QKRKRKKSRYKY & $280 \pm 13$ \\
18 & RKRKRKKSRKKH & $283 \pm 9$ \\
19 & KKRKRKKSRKRK & $315 \pm 11$ \\
14 & QFRKRKKSRYKF & $323 \pm 17$ \\
20 & QKRKRKKSRYRK & $405 \pm 20$ \\
16 & QHKRKRKKSRIVL & $424 \pm 37$ \\
8 & QKRKRKKSRYPLS & $542 \pm 16$ \\
6 & QKRKRKKSRYKK & $571 \pm 40$ \\
2 & QKRKRKKSRYPK & $602 \pm 19$ \\
12 & IVFKRKRKKSRYLS & $637 \pm 25$ \\
7 & QFRKRKKSRYKS & $665 \pm 38$ \\
13 & QFRKRKKSRYLS & $694 \pm 29$ \\
3 & QFRKRKKSRYPS & $4206 \pm 76$ \\
\hline & &
\end{tabular}



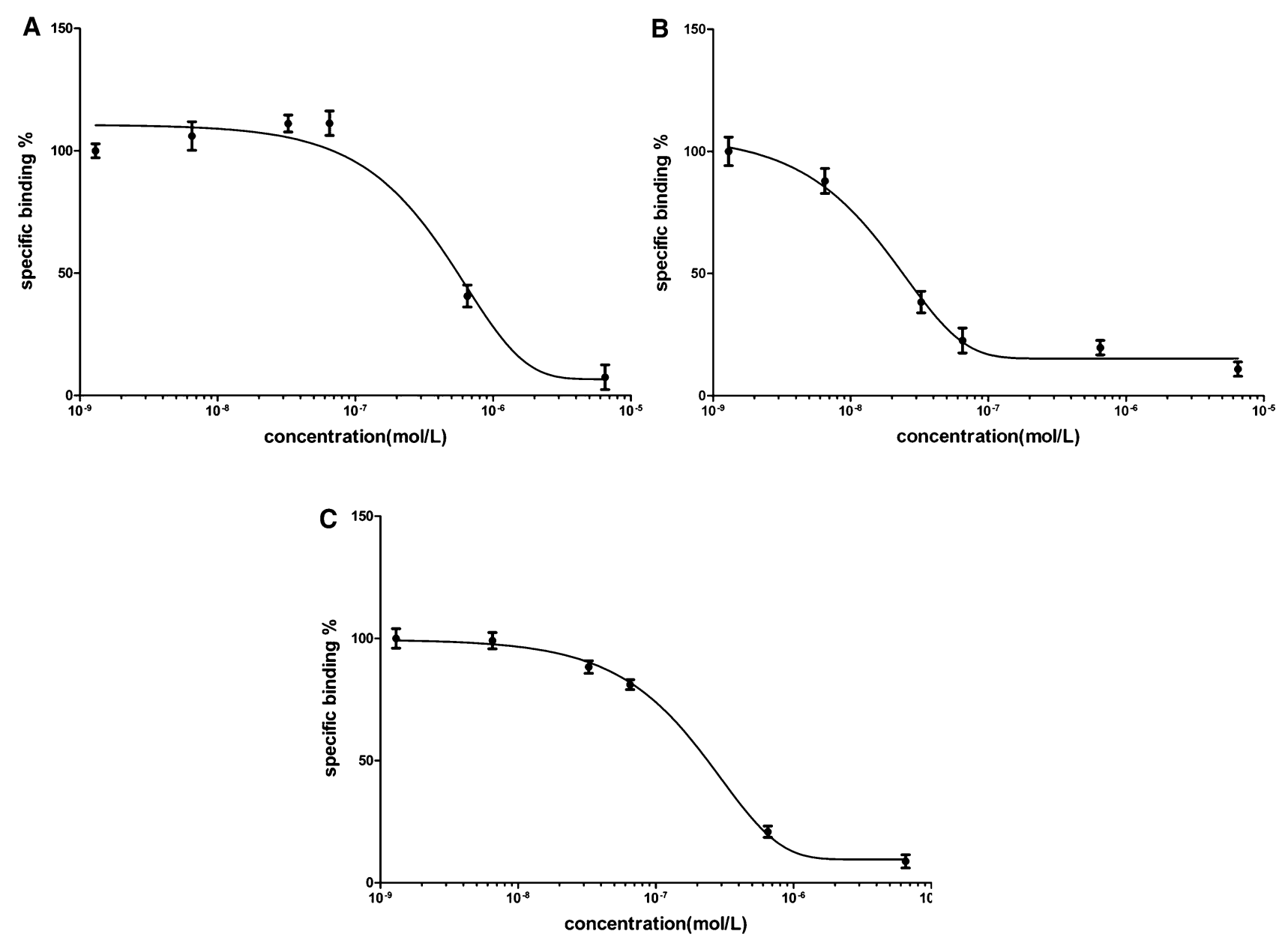

Fig. 4 VEGFR peptide binding inhibition curves. Analysis of ${ }^{125} \mathrm{I}-$ VEGF binding in the presence of $\operatorname{VEGF}_{125-136}(\mathbf{a})$, QKRKRKK SRKKH (b), or RKRKRKKSRYIVLS (c) using A549 cells resulted in

\section{Discussion}

Angiogenesis is an essential process involved in normal tissue development as well as for carcinogenesis of solid tumors and metastasis. VEGF plays a critical role in tumor angiogenesis and blocking VEGF/VEGFR signaling significantly inhibits tumor growth and metastasis $[13,23$, 24]. Thus, targeting VEGF/VEGFR with molecular probes holds great promise for use in radionuclide imaging or therapeutic applications.

An important criterion for effective polypeptide targeting is the affinity between a polypeptide and its targeted receptor, where higher affinity results in increased specificity and stronger targeting capability. Higher affinity binding produces decreased dissociation of the peptide from its receptor, resulting in higher tissue peptide concentrations with longer residency times. Our previous work showed that VEGF $_{125-136}$ exhibits good tumor-targeting properties [20]. In this study, we aimed to identify peptides
$\mathrm{IC}_{50}$ values of $464,80,185 \mathrm{nmol} / \mathrm{L}$, respectively. The $\mathrm{IC}_{50}$ value of each peptide was calculated using the SPSS13.0 statistics software package

with higher VEGFR-binding affinity with enhanced tumortargeting effects that could be used in molecular imaging and to substantially inhibit the growth of tumor cells or tumor vasculature endothelial cells.

Bioinformatic approaches enable the prediction and in silico selection of large numbers of candidate sequences at a relatively low cost and higher efficiency than through conventional biological experimentation. Molecular design methods employing the structure and interaction of biomacromolecules are gradually gaining ground in the study of polypeptides and drug design. Exploiting molecular docking and surface group interaction analyses, we designed 20 polypeptides with theoretically higher VEGFR-binding affinity than VEGF $_{125-136}$. As verified using biological methods, polypeptides selected using molecular docking had lower binding affinities than those identified by surface group interaction analysis; i.e., the predictive value of the former method is less optimal than the latter. This is likely to be due to the instability of polypeptide structures, as well as 

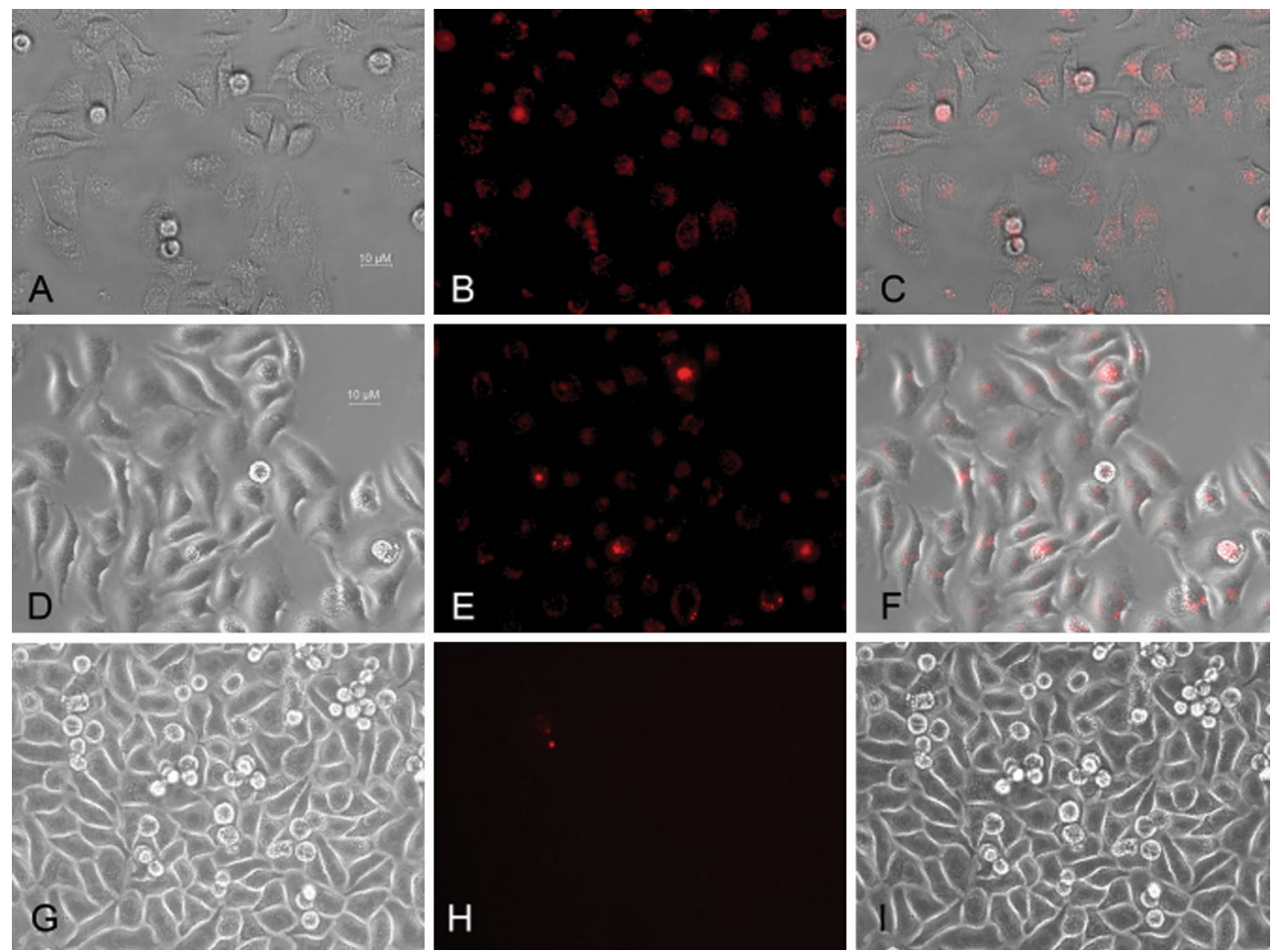

Fig. 5 Binding of rhodamine-conjugated peptides to A549 cells in vitro Rhodamine-labeled peptides were incubated with A549 cells for $6 \mathrm{~h}$ after which bright field (a QKRKRKKSRKKH, d RKRKRK KSRYIVLS, g rhodamine),sssss and red-fluorescent (b QKRKRKK

the complexity and vast number of calculations required for molecular docking analysis. In future studies, we plan to modify the molecular docking approach employed here and to perform a thorough molecular dynamics simulation to further improve the validity of molecular docking data in predicting and assessing the interaction between polypeptides and their receptors.

Using our bioinformatic strategy, we ultimately obtained 20 peptide candidates. Since the binding characteristics of these peptides were calculated in silico, biological experiments were required to verify the accuracy of the predictions and to positively identify the optimal peptide(s). Thus, competitive-binding assays were employed to determine the actual $\mathrm{IC}_{50}$ values with A549 cells. A549 cells are a human lung adenocarcinoma cell line that expresses high-levels of VEGFRs. These experiments used ${ }^{125} \mathrm{I}_{-} \mathrm{VEGF}_{165}$ as the standard protein, which has the greatest binding affinity for VEGFR. The observation of competitive binding with $\mathrm{VEGF}_{165}$ indicates that the peptide candidates had the capacity to bind the VEGFR. Using this method, we found that the computer predictions were not completely consistent with the biological experimental results. Peptides QKRKRKKSRKKH (NO.15) and RKRKRKKSRYIVLS (NO.17) exhibited the lowest $\mathrm{IC}_{50}$ values in competitive binding to VRGFR, which indicates that these two peptides had the highest binding affinities.
SRKKH, e RKRKRKKSRYIVLS, $\mathbf{h}$ unconjugated-rhodamine) images were obtained; merged images (c QKRKRKKSRKKH, f RKRKRKKS RYIVLS, i unconjugated-rhodamine) were generated at a $400 \times$ magnification. Unconjugated rhodamine was used as the negative control

Visualization of the cellular localization of peptide-binding sites was performed using fluorochrome-conjugated peptide staining of A549 cells. A549 cells were incubated with rhodamine-conjugated peptides and observed with fluorescence microscopy. These experiments indicated that labeling was observed mainly on the plasma membrane and in the perinuclear cytoplasm of A549 cells. These results are consistent with peptides targeting cell-surface receptors as well as undergoing intracellular transport through receptor-mediated internalization. Conversely, no significant fluorescent staining was observed in the control group, which used unconjugated rhodamine. This finding indicates that the peptides designed herein specifically bound VEGF receptors on A549 cells.

The polypeptides QKRKRKKSRKKH and RKRKRKK SRYIVLS exhibited $\approx 6$ - and 2.5-fold higher VEGFRbinding affinity than $\mathrm{VEGF}_{125-136}$, respectively. This finding suggests that these two peptides could be used as novel therapeutic agents or as molecular probes for tumortargeted radionuclide imaging.

\section{Conclusion}

Bioinformatic methods were successfully employed to identify two potential tumor-targeting small-molecule peptides with higher affinity for VEGFR than VEGF $_{125-136}$. 
This study establishes the experimental basis for further research into VEGFR targeting of tumors for molecular imaging and therapy.

Acknowledgments We thank Professor Shao-jun Cheng and postgraduate Lin Zha for their support. There are no potential conflicts of interest relevant to this article. This study was supported by the National Natural Science Foundation of China (30970848, 81171373)

Open Access This article is distributed under the terms of the Creative Commons Attribution License which permits any use, distribution, and reproduction in any medium, provided the original author(s) and the source are credited.

\section{References}

1. Khosravi, S. P., \& Fernández, P. I. (2008). Tumoral angiogenesis: Review of the literature. Cancer Investigation, 26, 104-108.

2. Keedy, V. L., \& Sandler, A. L. (2007). Inhibition of angiogenesis in the treatment of non-small cell lung cancer. Cancer Science, 98, 1825-1830.

3. Jia, B., Liu, Z., Shi, J., et al. (2008). Linker effects on biological properties of 111In-labeled DTPA conjugates of a cyclic RGDfK dimer. Bioconjugate Chemistry, 19, 201-210.

4. Liu, Z., Jia, B., Zhao, H., Chen, X., \& Wang, F. (2011). Specific targeting of human integrin $\alpha(\mathrm{v}) \beta$ (3) with (111)In-labeled Abegrin $^{\mathrm{TM}}$ in nude mouse models. Molecular Imaging and Biology, $13,112-120$.

5. Zhao, R., Yang, W., Wang, Z., Li, G., Qin, W., \& Wang, J. (2010). Treatment of transplanted tumor of lung adenocarcinoma A549 transfected by human somatostatin receptor subtype 2 (hsstr2) gene with ${ }^{188} \mathrm{Re}-\mathrm{RC}-160$. Nuclear Medicine and Biology, 37, 977-987.

6. Zhang, K., Aruva, M. R., Shanthly, N., et al. (2007). Vasoactive intestinal peptide (VIP) and pituitary adenylate cyclase activating peptide (PACAP) receptor specific peptide analogues for PET imaging of breast cancer: In vitro/in vivo evaluation. Regulatory Peptides, 144, 91-100.

7. Kim, H., Zhai, G., Liu, Z., et al. (2011). Extracellular matrix metalloproteinase as a novel target for pancreatic cancer therapy. Anti-Cancer Drugs, 22, 864-874.

8. Liu, Z. J., Tian, R., Li, Y., et al. (2011). Inhibition of tumor angiogenesis and melanoma growth by targeting vascular E-selectin. Annals of Surgery, 254, 450-456.

9. Wood, L. M., Pan, Z. K., Guirnalda, P., Tsai, P., Seavey, M., \& Paterson, Y. (2011). Targeting tumor vasculature with novel Listeria-based vaccines directed against CD105. Cancer Immunology, Immunotherapy, 60, 931-42.

10. Choe, Y. S., \& Lee, K. H. (2007). Targeted in vivo imaging of angiogenesis: Present status and perspectives. Current Pharmaceutical Design, 13, 17-31.
11. Trinh, X. B., Tjalma, W. A., Vermeulen, P. B., et al. (2009). The VEGF pathway and the AKT/mTOR/p70S6K1 signalling pathway in human epithelial ovarian cancer. British Journal of Cancer, 100, 971-978.

12. Park, M. S., Ravi, V., \& Araujo, D. M. (2010). Inhibiting the VEGF-VEGFR pathway in angiosarcoma, epithelioid hemangioendothelioma, and hemangiopericytoma/solitary fibrous tumor. Current Opinion in Oncology, 22, 351-355.

13. Sallinen, H., Anttila, M., Narvainen, J., et al. (2009). Antiangiogenic gene therapy with soluble VEGFR-1, -2 , and -3 reduces the growth of solid human ovarian carcinoma in mice. Molecular Therapy, 17, 278-284.

14. Ivy, S. P., Wick, J. Y., \& Kaufman, B. M. (2009). An overview of small-molecule inhibitors of VEGFR signaling. Nature Reviews Clinical Oncology, 6, 569-579.

15. Hsu, A. R., Cai, W., Veeravagu, A., et al. (2007). Multimodality molecular imaging of glioblastoma growth inhibition with vasculature-targeting fusion toxin VEGF121/rGel. Journal of Nuclear Medicine, 48, 445-454.

16. Backer, M. V., Levashova, Z., Patel, V., et al. (2007). Molecular imaging of VEGF receptors in angiogenic vasculature with single-chain VEGF-based probes. Nature Medicine, 13, 504-509.

17. Blankenberg, F. G., Backer, M. V., Levashova, Z., Patel, V., \& Backer, J. M. (2006). In vivo tumor angiogenesis imaging with site-specific labeled (99 m)Tc-HYNIC-VEGF. European Journal of Nuclear Medicine and Molecular Imaging, 33, 841-848.

18. Jia, H., Jezequel, S., Löhr, M., et al. (2001). Peptides encoded by exon 6 of VEGF inhibit endothelial cell biological responses and angiogenesis induced by VEGF. Biochemical and Biophysical Research Communications, 283, 164-173.

19. Bainbridge, J. W., Jia, H., Bagherzadeh, A., Selwood, D., Ali, R. R., \& Zachary, I. (2003). A peptide encoded by exon 6 of VEGF (EG3306) inhibits VEGF-induced angiogenesis in vitro and ischaemic retinal neovascularisation in vivo. Biochemical and Biophysical Research Communications, 302, 793-799.

20. Qin, Z. X., Li, Q. X., Liu, G. Y., et al. (2009). Imaging targeted at tumor with 188Re labeled VEGF189 exon 6 encoded peptide and effects of the transfecting truncated KDR gene in tumor-bearing nude mice. Nuclear Medicine and Biology, 36, 535-543.

21. Trott, O., \& Olson, A. J. (2010). AutoDock Vina: Improving the speed and accuracy of docking with a new scoring function, efficient optimization and multithreading. Journal of Computational Chemistry, 31, 455-461.

22. Petsalaki, E., Stark, A., García-Urdiales, E., \& Russell, R. B. (2009). Accurate prediction of peptide binding sites on protein surfaces. PLoS Computational Biology, 5(3), e1000335.

23. Shibuya, M., \& Claesson-Welsh, L. (2006). Signal transduction by VEGF receptors in regulation of angiogenesis and lymphangiogenesis. Experimental Cell Research, 312, 549-560.

24. Ma, Y. P., Yang, Y., Zhang, S., et al. (2010). Efficient inhibition of lung cancer in murine model by plasmid-encoding VEGF short hairpin RNA in combination with low-dose DDP. Journal of Experimental \& Clinical Cancer Research, 29, 56. 